\title{
Indices of Resting Metabolic Rate Accurately Reflect Energy Deficiency in Exercising Women
}

\author{
Nicole C.A. Strock, Kristen J. Koltun, Emily A. Southmayd, Nancy I. Williams, and Mary Jane De Souza \\ Penn State University
}

\begin{abstract}
Energy deficiency in exercising women can lead to physiological consequences. No gold standard exists to accurately estimate energy deficiency, but measured-to-predicted resting metabolic rate (RMR) ratio has been used to categorize women as energy deficient. The purpose of the study was to (a) evaluate the accuracy of RMR prediction methods, (b) determine the relationships with physiological consequences of energy deficiency, and (c) evaluate ratio thresholds in a cross-sectional comparison of ovulatory, amenorrheic, or subclinical menstrual disturbances in exercising women $(n=217)$. Dual-energy X-ray absorptiometry (DXA) and indirect calorimetry provided data on anthropometrics and energy expenditure. Harris-Benedict, DXA, and Cunningham (1980 and 1991) equations were used to estimate RMR and RMR ratio. Group differences were assessed (analysis of variance and Kruskal-Wallis tests); logistic regression and Spearman correlations related ratios with consequences of energy deficiency (i.e., low total triiodothyronine; $\mathrm{TT}_{3}$ ). Sensitivity and specificity calculations evaluated ratio thresholds. Amenorrheic women had lower RMR $(p<.05)$, DXA ratio $(p<.01)$, Cunningham $_{1980}(p<.05)$ and Cunningham $_{1991}(p<.05)$ ratio, and TT $_{3}(p$ $<.01)$ compared with the ovulatory group. Each prediction equation overestimated measured RMR $(p<.001)$, but predicted $(p<.001)$ and positively correlated with $\mathrm{TT}_{3}(r=.329-.453)$. A 0.90 ratio threshold yielded highest sensitivity for Cunningham $_{1980}(0.90)$ and Harris-Benedict (0.87) methods, but a higher ratio threshold was best for DXA (0.94) and Cunningham 1991 (0.92) methods to yield a sensitivity of 0.80 . In conclusion, each ratio predicted and correlated with $\mathrm{TT}_{3}$, supporting the use of RMR ratio as an alternative assessment of energetic status in exercising women. However, a 0.90 ratio cutoff is not universal across RMR estimation methods.
\end{abstract}

Keywords: female athlete triad, menstrual disturbances, metabolic suppression

Energy deficiency in exercising women is associated with severe consequences including reproductive dysfunction (De Souza et al., 2007b; Williams et al., 2015) and impaired bone health (De Souza et al., 2008), a condition referred to as the Female Athlete Triad (Triad) (De Souza et al., 2014; Nattiv et al., 2007). When energy intake fails to meet metabolic demands, metabolizable fuels are repartitioned toward the physiological processes necessary for survival (i.e., locomotion, cellular maintenance, and thermoregulation) and away from those energetic processes deemed unnecessary for survival (i.e., growth and reproduction). This results in suppression of metabolism, energy expenditure, and thyroid hormones (Wade et al., 1996), contributing to the development of exercise-associated menstrual disturbances (Loucks et al., 1998). Exercise-associated menstrual disturbances can range in severity from luteal phase defects to amenorrhea consistent with the magnitude of energy deficiency, such that those with the most severe menstrual disturbances are also the most energy deficient (De Souza et al., 2007b). Owing to the long-term health outcomes associated with energy deficiency (De Souza et al., 2007b, 2008; Williams et al., 2015), early intervention and prevention are necessary.

Currently, energy deficiency has no units of measure and there is no standard of measurement, thereby requiring alternative methods to be used for its identification. At present, the calculation of energy availability, defined as dietary intake minus exercise

The authors are with the Women's Health and Exercise Laboratory, Department of Kinesiology, Penn State University, University Park, PA. De Souza (mjd34@psu. edu) is corresponding author. energy expenditure (Loucks \& Heath, 1994), has been utilized to assess energetic status in exercising women (Lieberman et al., 2018; Loucks et al., 1998; Reed et al., 2015; Williams et al., 2015); however, concerns exist regarding the accurate calculation of energy availability, thereby highlighting the necessity for development and validation of an accurate and accessible method to identify energy deficiency.

Metabolic suppression occurs when energy intake and body weight are reduced (Leibel et al., 1995). Based on this, our laboratory has utilized the ratio of measured resting metabolic rate $(\mathrm{RMR})$ to predicted $\mathrm{RMR}$ based on prediction equations to identify those at risk for being energy deficient (De Souza et al., 2007a, 2008; Scheid et al., 2009). Comparisons of measured-topredicted RMR were initially utilized to characterize the energy deficiency in patients with anorexia (Melchior et al., 1989; Vaisman et al., 1988) and, as such, we reasoned that this strategy may also be applicable in exercising women to identify energy deficiency. In particular, we have suggested that an $\mathrm{RMR}$ ratio $\leq 0.90$, representing a measured value that is $90 \%$ of the predicted value, may be indicative of metabolic suppression secondary to energy deficiency in the population of exercising women. A $\leq 0.90$ ratio has been supported in the literature and associated with other proxy indicators of energy deficiency including high drive for thinness (De Souza et al., 2007a; Gibbs et al., 2011), high cognitive restraint (Vescovi et al., 2008), low energy availability as assessed by the Low Energy Availability in Females Questionnaire (Staal et al., 2018), low total triiodothyronine $\left(\mathrm{TT}_{3}\right.$ ) (De Souza et al., 2008), high peptide YY (Scheid et al., 2009), measured low energy availability (Melin et al., 2015), and menstrual disturbances (Gibbs et al., 2011). 
Currently, a variety of prediction equations are available in the literature and have been used to estimate RMR and calculate RMR ratio in exercising women (Cunningham, 1980, 1991; Harris \& Benedict, 1918; Hayes et al., 2002). The initial ratio was developed for use with the Harris-Benedict equation (De Souza et al., 2007b, 2008; Reed et al., 2015; Scheid et al., 2009; Vanheest et al., 2014; Vescovi et al., 2008), although others have reported that the Cunningham 1980 equation may yield a more accurate estimate of RMR among highly active individuals (Thompson \& Manore, 1996), particularly because of higher lean body mass and fat-free mass. Interestingly, two different Cunningham equations exist (1980 and 1991), one of which relies on lean body mass and the other on fat-free mass. However, there are several examples of researchers using fat-free mass in the calculation for the Cunningham ${ }_{1980}$ equation (Jagim et al., 2018; Staal et al., 2018; ten Haaf \& Weijs, 2014; Thompson \& Manore, 1996; Tinsley et al., 2019), a method that was developed to specifically use lean body mass to estimate RMR (Cunningham, 1980). Whether or not the use of lean body mass and fat-free mass interchangeably may impact the result of predicted RMR and RMR ratio is unknown. Most recently, dual-energy X-ray absorptiometry (DXA) modeling, based on tissue-specific metabolic rates (Gallagher et al., 1998; Hayes et al., 2002), may represent a unique and more accurate depiction of metabolic rate and has been applied to exercising women (Koehler et al., 2016). Because there are several equations in use for exercising women and as different prediction equations may provide variable estimates of metabolic rate (Staal et al., 2018), it is necessary to assess the ability of each ratio to predict energy deficiency and evaluate the appropriateness of the ratio $\leq 0.90$ as an indicator of energy deficiency.

Thus, the purpose of this study was to (a) evaluate values from Harris-Benedict, Cunningham (1980 and 1991), and DXA prediction methods against indirect calorimetry-measured RMR; (b) determine how each ratio relates to known physiological consequences of energy deficiency; and (c) confirm the use of a 0.90 ratio or propose alternative threshold cutoff values for use with each of the prediction equations to estimate energy deficiency.

\section{Methods}

\section{Design}

This study is a cross-sectional secondary analysis of exercising women $(n=217)$, representing a spectrum of energetic and reproductive statuses. The data set is derived from three studies (Figure 1), which were conducted using identical procedures at the University of Toronto and Penn State University directed by the same principal investigator (M.J. De Souza) to assess women's health, exercise, and menstrual function. The following data sets were used given their methodological similarities: (a) baseline data from a 12-month randomized controlled trial to assess the impact of increased energy intake on reproductive function and bone health (Study 1: REFUEL, $n=116$; Williams et al., 2019); (b) data from a crosssectional observational study at University of Toronto (Study 2A: $n=50$; O’Donnell et al., 2007) and an ongoing study at Penn State

\section{Potential participants $(n=427)$}

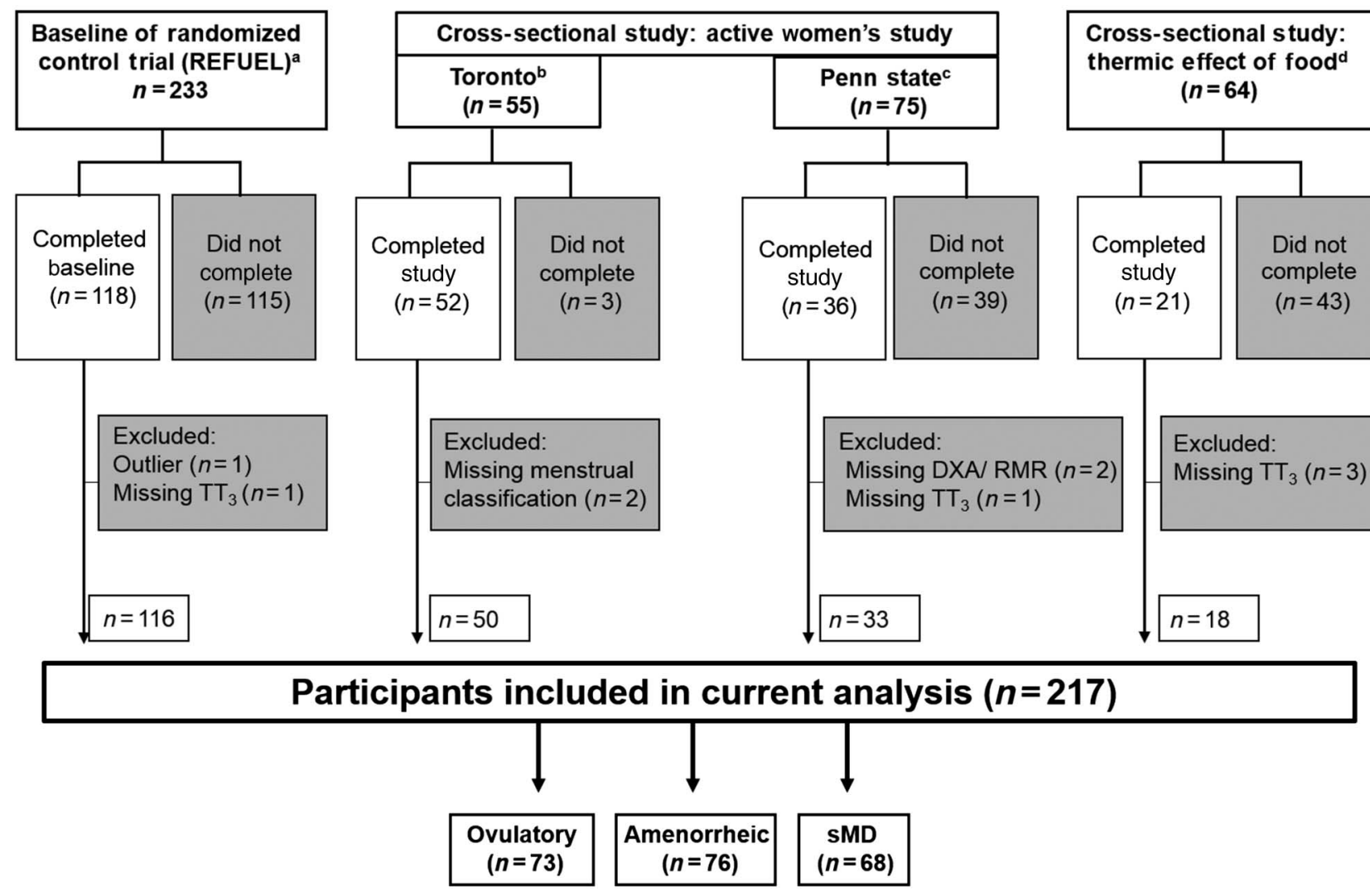

Figure 1 - Inclusion and exclusion criteria for current study. $\mathrm{TT}_{3}=$ total triiodothyronine; $\mathrm{DXA}=$ dual-energy X-ray absorptiometry; RMR = resting metabolic rate. ${ }^{\mathrm{a}}$ Study 1 (Williams et al., 2019). ' Study 2A (O'Donnell et al., 2007). ${ }^{\mathrm{c}}$ Study $2 \mathrm{~B}$ (Southmayd et al., 2019). ${ }^{\mathrm{d}}$ Study 3 (Koltun et al., 2019). 
University (Study 2B: $n=33$; Southmayd et al., 2019) evaluating the relationship of metabolism, reproductive status, and bone health in exercising women; and (c) a cross-sectional study investigating metabolic and gut hormone responses to ingested meal (Study 3: $n=18$; Koltun et al., 2019). Inclusion and exclusion criteria for these studies were similar thus warranting pooling of data. Small differences in age range (Studies 1 and 3: 18-35 years; Study 2: 18-30 years), body mass index (Study $1: 16-25 \mathrm{~kg} / \mathrm{m}^{2}$; Study 2: $16-29.9 \mathrm{~kg} / \mathrm{m}^{2}$; Study 3: $16-35 \mathrm{~kg} / \mathrm{m}^{2}$ ), and weight stability (Studies 1 and 3: weight stable within $2 \mathrm{~kg}$ for 6 months; Study 2: no requirement) were not of sufficient magnitude to introduce bias into the current analysis. Each participant in this analysis participated in only one of the three studies. Inclusion criteria encompassing all studies are detailed in the "Participants" section. To be included in the analysis, women had to complete daily urine collection over the course of one menstrual cycle if eumenorrheic or one 28-day monitoring period if not regularly cycling to compare reproductive and energetic profiles. Additionally, women were excluded if they did not have measurements needed to assess metabolism (RMR, DXA, and serum $\mathrm{TT}_{3}$ ).

\section{Participants}

Volunteers for each study were recruited via campus flyers, classroom announcements, and newspaper and Internet advertisements. Screening procedures included questionnaires on exercise history, eating behaviors, menstrual history, and self-reported medical health history. Inclusion criteria were as follows: (a) age 18-35 years, (b) body mass index $16-35 \mathrm{~kg} / \mathrm{m}^{2}$, (c) $\geq 2 \mathrm{hr}$ of purposeful moderate- to vigorous-intensity exercise per week, (d) nonsmoking, (e) no medication use that would alter metabolic or reproductive hormone concentrations, and (f) no current clinical diagnosis of an eating or psychiatric disorder. Women were excluded if they had any contraindicated health condition, were taking hormonal contraception or medications that would affect metabolism, had current clinical diagnosis of eating or psychiatric disorder, or had known organic causes of menstrual disorders. Each study received ethical approval by the University of Toronto and Penn State University Institutional Review Boards (Koltun et al., 2019; O'Donnell et al., 2007; Southmayd et al., 2019; Williams et al., 2019). Each participant was informed of the purpose, procedures, and potential risks of participation in the study before signing an institutional review board-approved informed consent.

\section{Group Categorization}

Participants were grouped after determination of menstrual status based on daily urinary profiles of reproductive hormones as follows: (a) exercising eumenorrheic women with ovulatory menstrual cycles and no luteal phase defects $(n=73)$, (b) exercising amenorrheic women $(n=76)$, and (c) exercising women with subclinical menstrual disturbances (sMD) including oligomenorrhea, anovulation, and luteal phase defects $(n=68)$.

\section{Menstrual Characteristics}

Menstrual status was based on self-reported menstrual history and confirmed by measurements of daily urinary reproductive hormone metabolites (estrone-1-glucuronide [E1G] and pregnanediol glucuronide $[\mathrm{PdG}]$ ) for one complete menstrual cycle in ovulatory and sMD groups or a 28-day monitoring period in amenorrheic group.
Urinary measurements of E1G and PdG have been previously described (De Souza et al., 2010). Women were considered ovulatory if they reported and demonstrated a menstrual cycle length between 26 and 35 days (De Souza et al., 1998, 2010) and met criteria for detectable ovulation from urinary assays (De Souza et al., 2010; Santoro et al., 2003). Volunteers were considered amenorrheic if they failed to menstruate for a minimum of three consecutive months or oligomenorrheic if menses occurred at irregular intervals of 36-90 days (De Souza et al., 1998, 2010). Quantification of luteal phase defects and anovulatory cycles has been previously described (De Souza et al., 1998, 2010; Santoro et al., 2003). Oligomenorrheic cycles were assessed for the duration of the intermenstrual interval, for daily E1G and PdG concentrations, and to determine the presence or absence of an ovulatory event, as defined earlier and previously reported (De Souza et al., 2010). Menstrual cycle length was defined as the number of days from Day 1 of menses to the day before the first day of the next menses (De Souza et al., 1998, 2010).

\section{Anthropometrics and DXA}

Total body mass was measured weekly to the nearest $0.1 \mathrm{~kg}$ on a physician's scale (Seca Model 770; Seca, Hamburg, Germany), and height was measured by stadiometer to the nearest $0.5 \mathrm{~cm}$ at the beginning of the study. Body mass index was calculated as weight divided by height squared $\left(\mathrm{kg} / \mathrm{m}^{2}\right)$.

Percentage body fat, fat mass, fat-free mass, and lean body mass were assessed using DXA. An International Society of Clinical Densitometry-certified technician performed all DXA scans. Participants were scanned on a Lunar Prodigy DXA scanner (enCORE 2002 software, version 6.50.069; General Electric, Madison, WI; $n=87$ ), Lunar iDXA scanner (enCORE 2008 software, version 12.10.113; General Electric; $n=117$ ), or Hologic QDR4500W DXA scanner (Hologic, Bedford, MA; $n=13$ ). Cross-calibration studies consistent with the guidelines of the International Society for Clinical Densitometry were performed to remove bias and have been previously described (Koehler et al., 2016). Using data from the DXA analyses, organ tissue mass of the brain, skeletal muscle, bone, adipose tissue, and residual mass were determined as outlined by Hayes et al. (2002), using published tissue-specific metabolic rates to determine DXA-predicted RMR (Elia, 1992), and as previously described (Koehler et al., 2016).

\section{Determination of RMR and RMR Ratio}

RMR was measured to assess energetic status following a 12-hr fast and $24 \mathrm{hr}$ of no exercise or caffeine, during the early follicular phase (Days 2-6) of the monitoring period for menstruating participants or during the first 6 days of the 28-day monitoring period for amenorrheic participants. RMR measurements were performed on the participants between 0800 and $1000 \mathrm{hr}$ in a supine position for a minimum of $30 \mathrm{~min}$, after a 30- to 45-min rest period. RMR was assessed via indirect calorimetry using a ventilated hood (SensorMedics Vmax Series; CareFusion, Yorba Linda, CA) as previously described (De Souza et al., 2008; Scheid et al., 2009). Four prediction equations, commonly used in an exercising population, were utilized to estimate RMR: (a) Harris-Benedict equation (Harris \& Benedict, 1918); (b) DXA equation (Hayes et al., 2002; Koehler et al., 2016); (c) Cunningham 1980 equation, which utilizes lean body mass (Cunningham, 1980); and (d) Cunningham 1991 equation, which utilizes fat-free mass (Cunningham, 1991). 
For each prediction equation, a ratio of measured-to-predicted RMR values was calculated. The rationale for 0.90 ratio cutoff was based from comparisons of measured and predicted RMR in women with anorexia nervosa (Melchior et al., 1989; Vaisman et al., 1988), a model presumed to be associated with more severe energy deficiency than exercising women without an eating disorder (De Souza et al., 2007a, 2008). Measured RMR in anorexics was typically between $49 \%$ and $91 \%$ of Harris-Benedict predicted values (Melchior et al., 1989; Vaisman et al., 1988), corresponding to RMR ratio of $0.49-0.91$. We have previously reported HarrisBenedict ratio $\leq 0.90$ to be risk factors and markers of energy deficiency including high drive for thinness (De Souza et al., 2007a), $0.88 \pm 0.02$ in exercising amenorrheic women (Scheid et al., 2009), $0.87 \pm 0.01$ in energy- and estrogen-replete women, and $0.80 \pm 0.15$ in energy- and estrogen-deficient women (De Souza et al., 2008). Alternatively, sedentary and ovulatory exercising women both presented with a ratio of $0.94 \pm 0.02$ (Scheid et al., 2009). As such, a threshold cutoff of 0.90 was reasoned to be useful, with values $>0.90$ indicating energy-replete status and values $\leq 0.90$ indicating an energy-deficient status when utilizing the Harris-Benedict equation. To date, several research papers have been published that have utilized a $\mathrm{RMR}$ ratio $\leq 0.90$ as a proxy for categorizing exercising women as energy deficient using the Harris-Benedict equation (De Souza et al., 2008; Gibbs et al., 2011; Scheid et al., 2009; Staal et al., 2018; Vescovi et al., 2008) and the Cunningham 1980 equation (Melin et al., 2015; Staal et al., 2018).

\section{Psychological Questionnaires}

Participants completed the Eating Disorder Inventory-2 (Garner, 1991) and Three-Factor Eating Questionnaire-R21 (Bond et al., 2001; Cappelleri et al., 2009) at screening to assess eating behaviors.

\section{Blood Sampling and Storage}

Blood samples were collected once between 0730 and $1000 \mathrm{hr}$ during the baseline measurement session for all volunteers. Volunteers were instructed not to exercise or consume food within $12 \mathrm{hr}$ prior to blood sampling. Antecubital blood samples were drawn using a blood collection needle (21 gauge, $19 \mathrm{~mm}$ ) and blood collection tubes (BD Vacutainer; Becton, Dickinson and Company, Franklin Lakes, NJ). Samples were allowed to clot for $30 \mathrm{~min}$ at room temperature $\left(20-24^{\circ} \mathrm{C}\right)$ and then centrifuged (Eppendorf centrifuge 5804 R; Eppendorf, Hamburg, Germany) for $15 \mathrm{~min}$ at $4{ }^{\circ} \mathrm{C}$. The serum was aliquoted into $2-\mathrm{ml}$ polyethylene storage tubes and frozen at $-80{ }^{\circ} \mathrm{C}$ until analysis.

\section{Serum Hormone Measurements}

Total triiodothyronine concentration was analyzed using a chemiluminescence immunoassay analyzer (Immulite; Siemens Healthcare, Erlangen, Germany) through competitive immunoassay. Analytical sensitivity for the $\mathrm{TT}_{3}$ assay was $0.54 \mathrm{nmol} / \mathrm{L}$ $(35 \mathrm{ng} / \mathrm{dl})$. The intraassay and interassay coefficients of variation were $8.2 \%$ and $10.6 \%$, respectively.

\section{Statistical Analysis}

Data screening was conducted prior to statistical analysis in order to identify whether the data met the assumptions required by specific statistical techniques. Data screening involved outlier detection ( $>3 S D$ s of mean) and examination of variable distributions within each of the three groups for normality. One-way analyses of variance were conducted on normally distributed data with Bonferroni post hoc tests to detect group differences (ovulatory vs. amenorrheic vs. sMD). Kruskal-Wallis tests were conducted on nonnormally distributed data to determine group differences. A repeated-samples Friedman's two-way analysis of variance was used to compare differences between RMR and Harris-Benedict, Cunningham (1980 and 1991), and DXApredicted RMR values. Logistic regression was used to determine if $\mathrm{RMR}$ ratio successfully predicted at-risk Triad conditions cited in the literature (De Souza et al., 2014; Nattiv et al., 2007) including amenorrhea, body mass index $<18.5 \mathrm{~kg} / \mathrm{m}^{2}$, drive for thinness $>7$, and low $\mathrm{TT}_{3}$. Low $\mathrm{TT}_{3}$ was determined based on the lowest tertile of $\mathrm{TT}_{3}$ values within this data set (low $\mathrm{TT}_{3}<1.12 \mathrm{nmol} / \mathrm{L}$ ), which captures values lower than the normal range of $\mathrm{TT}_{3}$ values (Morley et al., 1983), as well as those cited in amenorrheic (Thomson et al., 1977) and energy-deficient (Loucks \& Thuma, 2003; Williams et al., 1995) women. Calculations of sensitivity and specificity (Altman \& Bland, 1994) were used to compare RMR ratio determinations to evaluate efficacy of 0.90 ratio cutoff for each prediction method. Grading of sensitivity scores was determined a priori; acceptable sensitivity was scored as $\geq 0.80$ and good sensitivity was scored as $\geq 0.90$. Correlations were calculated using Spearman's correlation analysis for $\mathrm{TT}_{3}$ and nonnormally distributed RMR ratios. All statistical analysis was performed in IBM SPSS Statistics for Windows (version 25.0; IBM Corp., Armonk, NY). Data were expressed as mean $\pm S D$.

\section{Results}

\section{Demographics Characteristics}

Of the 427 women initially enrolled in the three studies, 210 were excluded because of a failure to complete baseline study procedures including one-cycle urine collection, DXA scan, and RMR procedure (Figure 1). Among the remaining 217 women, 73 were classified as ovulatory, 76 were classified as amenorrheic, and 68 were classified as sMD, which included participants with oligomenorrhea, anovulation, and luteal phase defects.

Demographic characteristics of the participants $(n=217)$ are presented in Table 1 . The ovulatory group was older compared with amenorrheic $(p<.001)$ and sMD groups $(p<.001)$. Ovulatory and sMD groups were similar with respect to body weight, but amenorrheic had lower body weight than SMD $(p<.01)$. The amenorrheic group had lower body mass index $(p=.007, p<.001)$, body fat percentage $(p=.005, p<.001)$, and fat mass $(p=.003, p<.001)$ than both ovulatory and SMD groups, respectively.

\section{Menstrual Characteristics}

Quantification of E1G and PdG metabolites in daily urine samples collected for one complete menstrual cycle or monitoring period are demonstrated as composite menstrual graphs for each group (Figure 2). Cycle length for ovulatory (29.6 \pm 3.1 days) and sMD (30.2 \pm 6.7 days) groups were comparable. Amenorrheic participants reported amenorrhea lasting 276 \pm 225 days prior to study participation. Similarly, amenorrheic participants had significantly lower estrogen $(p<.001)$ and progesterone $(p<.001)$ exposure, expressed as area under curve, than both ovulatory and SMD. 
Table 1 Subject Characteristics

\begin{tabular}{|c|c|c|c|c|}
\hline Variables & Ovulatory $(n=73)$ & Amenorrheic $(n=76)$ & sMD $(n=68)$ & $p$ value \\
\hline \multicolumn{5}{|l|}{ Subject characteristics } \\
\hline Age (years) & $24.6 \pm 4.6$ & $21.6 \pm 3.3^{*}$ & $21.7 \pm 3.5^{*}$ & $<.001$ \\
\hline Age of menarche & $12.6 \pm 1.3$ & $13.3 \pm 1.5^{*}$ & $13.1 \pm 1.8$ & .024 \\
\hline Gynecological age & $12.1 \pm 4.8$ & $8.4 \pm 3.6^{*}$ & $8.6 \pm 4.0^{*}$ & .000 \\
\hline Body mass $(\mathrm{kg})$ & $59.9 \pm 8.9$ & $56.7 \pm 7.0 * *$ & $60.2 \pm 7.0$ & .007 \\
\hline Body mass index $\left(\mathrm{kg} / \mathrm{m}^{2}\right)$ & $22.1 \pm 2.7$ & $20.7 \pm 2.2 * * * *$ & $21.9 \pm 1.9$ & .001 \\
\hline Body fat $(\%)$ & $27.4 \pm 5.7$ & $24.0 \pm 5.9 * * *$ & $26.4 \pm 4.6$ & .001 \\
\hline Fat mass $(\mathrm{kg})$ & $16.4 \pm 5.4$ & $13.6 \pm 4.3^{*} \cdot * *$ & $15.9 \pm 3.9$ & .001 \\
\hline Lean body mass (kg) & $40.9 \pm 5.4$ & $40.7 \pm 4.7$ & $41.8 \pm 4.7$ & .352 \\
\hline Fat-free mass $(\mathrm{kg})$ & $43.4 \pm 5.6$ & $42.9 \pm 4.8$ & $44.2 \pm 4.7$ & .356 \\
\hline \multicolumn{5}{|l|}{ Metabolic characteristics } \\
\hline RMR (kcal/day) & $1,234 \pm 169$ & $1,169 \pm 175^{* * * *}$ & $1,236 \pm 148$ & .015 \\
\hline Harris-Benedict RMR (kcal/day) & $1,410 \pm 90$ & $1,402 \pm 78 * *$ & $1,436 \pm 77$ & .042 \\
\hline DXA RMR (kcal/day) & $1,282 \pm 139$ & $1,302 \pm 130$ & $1,310 \pm 126$ & .573 \\
\hline Cunningham $_{1980}$ RMR (kcal/day) & $1,400 \pm 120$ & $1,395 \pm 103$ & $1,420 \pm 103$ & .352 \\
\hline Cunningham $_{1991}$ RMR (kcal/day) & $1,307 \pm 123$ & $1,298 \pm 103$ & $1,324 \pm 102$ & .356 \\
\hline Harris-Benedict ratio & $0.88 \pm 0.11$ & $0.83 \pm 0.10$ & $0.86 \pm 0.09$ & .066 \\
\hline DXA ratio & $0.96 \pm 0.10$ & $0.90 \pm 0.11^{*} * *$ & $0.95 \pm 0.10$ & .001 \\
\hline Cunningham $_{1980}$ ratio & $0.88 \pm 0.09$ & $0.84 \pm 0.09 *$ & $0.87 \pm 0.09$ & .017 \\
\hline Cunningham $_{1991}$ ratio & $0.94 \pm 0.09$ & $0.90 \pm 0.10^{*}$ & $0.94 \pm 0.09$ & .025 \\
\hline EA (kcal/kg fat-free mass/day) & $33.6 \pm 8.8$ & $34.6 \pm 15$ & $35.7 \pm 13$ & .791 \\
\hline \multicolumn{5}{|l|}{ Hormone characteristics } \\
\hline $\mathrm{TT}_{3}(\mathrm{nmol} / \mathrm{L})$ & $1.4 \pm 0.28$ & $1.23 \pm 0.36^{*} * *$ & $1.4 \pm 0.35$ & .004 \\
\hline E1G AUC (ng/ml) & $1,433 \pm 697$ & $784 \pm 566^{*, * *}$ & $1,419 \pm 795$ & $<.001$ \\
\hline PdG AUC $(\mu \mathrm{g} / \mathrm{ml})$ & $82.8 \pm 38$ & $30.2 \pm 23 * * *$ & $56.9 \pm 46^{*}$ & $<.001$ \\
\hline \multicolumn{5}{|l|}{ Psychological characteristics } \\
\hline EDI-Drive for thinness & $1.6 \pm 3.4$ & $3.7 \pm 4.4^{*}$ & $2.9 \pm 4.1 *$ & $<.001$ \\
\hline TFEQ_-Cognitive restraint & $7.8 \pm 4.8$ & $11.7 \pm 4.9^{*}$ & $10.1 \pm 5.3$ & $<.001$ \\
\hline
\end{tabular}

Note. Values given in mean $\pm S D$. Cunningham ${ }_{1980}, \mathrm{RMR}=500+22$ (lean body mass); Cunningham ${ }_{1991}, \mathrm{RMR}=370+21.6$ (fat-free mass). Conversion factor between $\mathrm{nmol} / \mathrm{L}$ and $\mathrm{ng} / \mathrm{dl}$ is 0.0153 . sMD = subclinical menstrual disturbance; RMR = resting metabolic rate; DXA = dual-energy X-ray absorptiometry; EA = energy availability; $\mathrm{TT}_{3}=$ total triiodothyronine; $\mathrm{E} 1 \mathrm{G}=$ estrone-1-glucuronide; $\mathrm{AUC}=$ area under curve; $\mathrm{PdG}=$ pregnanediol glucuronide; EDI = Eating Disorder Inventory; TFEQ = ThreeFactor Eating Questionnaire.

*Significantly different from ovulatory. **Significantly different from sMD. Bold values denote significance at $p<.05$.

\section{Energetic and Psychological Characteristics}

Energetic characteristics of the participants $(n=217)$ are presented in Table 1. The amenorrheic group had lower measured RMR compared with ovulatory $(p=.036)$ and $\operatorname{sMD}(p=.041)$, a lower Harris-Benedict RMR than the sMD group $(p=.045)$, and a lower Cunningham $_{1980}(p=.021)$ and Cunningham ${ }_{1991}(p=.034)$ ratios than the ovulatory group. Additionally, amenorrheic participants had a lower DXA ratio compared with both the ovulatory $(p=.002)$ and sMD groups $(p=.018)$. There were no group differences in energy availability. Each prediction method produced values that were different from measured RMR $(p<.001)$, and on average, DXA and Cunningham ${ }_{1991}$ predicted RMR values were $\sim 10 \%$ greater than measured RMR, compared with Cunningham 1980 and Harris-Benedict RMR values, which were $\sim 20 \%$ greater than measured RMR. Of the DXA and Cunningham 1991 predicted values, $48.8 \%$ and $49.3 \%$, respectively, were within $\pm 100 \mathrm{kcal}$ of measured RMR, while only $19.4 \%$ and $9.7 \%$ of Cunningham 1980 and Harris-Benedict predicted values were within the same range
(Figure 3). The amenorrheic group had the lowest $\mathrm{TT}_{3}$ hormone concentrations compared with both the ovulatory $(p=.007)$ and sMD groups $(p=.023)$. The ovulatory group had a lower drive for thinness compared with amenorrheic $(p<.001)$ and $\operatorname{sMD}(p=.030)$ groups.

\section{Relationship Between RMR Ratios and Indicators of Energy Deficiency}

Logistic regression was performed to determine if RMR ratio predicted conditions associated with the risk of the Triad (Table 2). Each prediction equation ratio yielded significant regression models for predicting amenorrhea (Harris-Benedict: $p=.013$; DXA: $p<.001$; Cunningham ${ }_{1980}: p=.003$; Cunningham ${ }_{1991}$; $p=.005$ ) and $\mathrm{TT}_{3}$ (all ratios: $p<.001$ ). Each regression model correctly identified approximately $70 \%$ of women with low $\mathrm{TT}_{3}$ and $65 \%$ of women with amenorrhea. Each RMR ratio was positively associated with the hormone marker of energetic status, 
(a) Ovulatory $(n=73)$

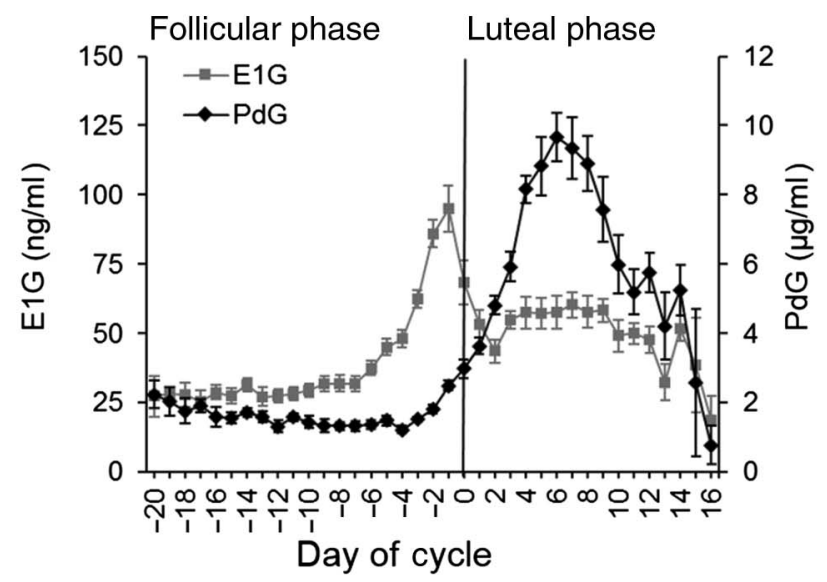

(c) Subclinical menstrual disturbances $(n=68)$

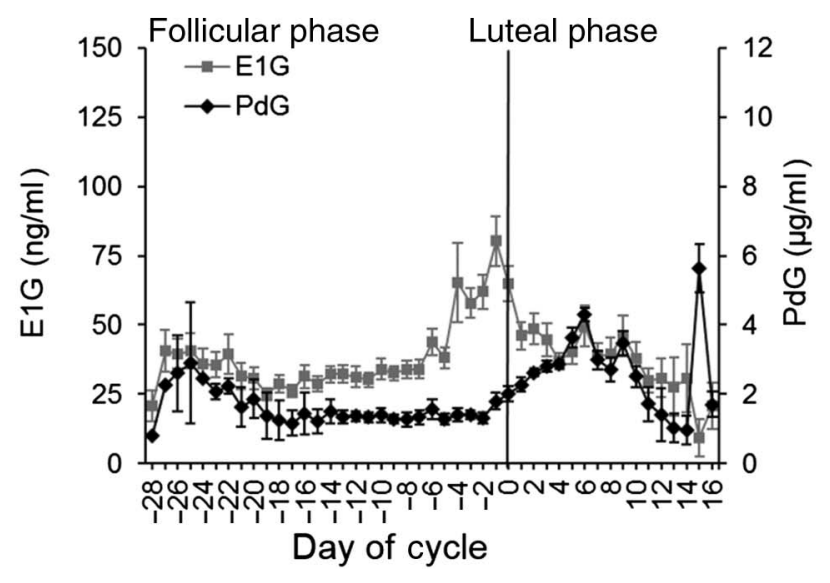

\section{(b) Amenorrheic $(n=76)$}

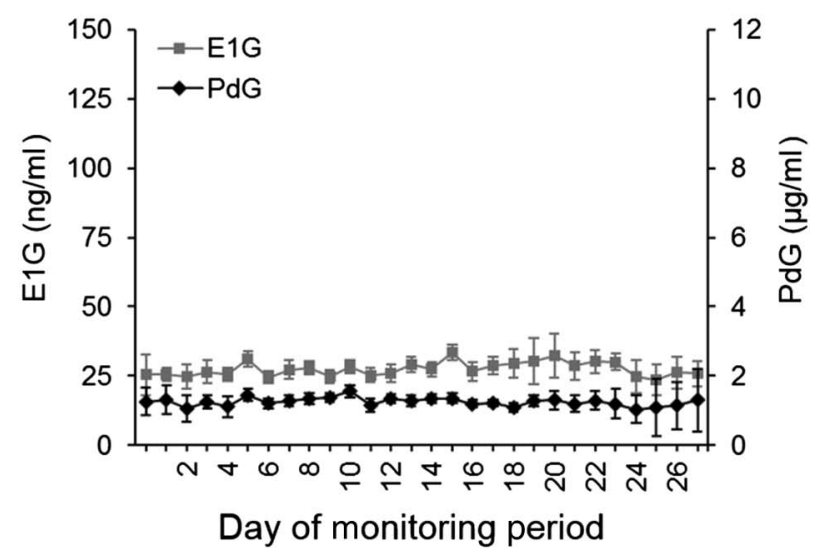

Figure 2 - Composite menstrual profiles for ovulatory, amenorrheic, and subclinical menstrual disturbance (sMD) groups. E1G=estrone-1glucuronide; $\mathrm{PdG}=$ pregnanediol glucuronide.

$\mathrm{TT}_{3}$ (DXA: $r=.453, p<.001 ;$ Cunningham $1980: r=.444, p<.001$; Cunningham $_{1991}: r=.447, p<.001$; Harris-Benedict: $r=.329$, $p<.001)$.

\section{Sensitivity and Specificity of RMR Ratio}

Using $\mathrm{TT}_{3}$ as an indicator of energy deficiency, sensitivity and specificity were calculated to determine appropriate threshold cutoff values for RMR ratio. First, a 0.90 ratio cutoff commonly used in Triad literature was evaluated for the detection of low $\mathrm{TT}_{3}$. Cunningham $_{1980}$ ratio correctly identified the most participants (sensitivity: 0.90), but had low specificity (0.41), and the Harris-Benedict ratio produced comparable sensitivity (0.87) and specificity (0.43). DXA and Cunningham ${ }_{1991}$ ratio had the lowest sensitivity scores (0.62 and 0.63 ) but correctly identified the participants without low $\mathrm{TT}_{3}$ (specificity: 0.74 and 0.72 ), at a 0.90 cutoff.

Because DXA and Cunningham 1991 values were closer to measured RMR and the resultant ratio yielded poor sensitivity at a 0.90 ratio cutoff, additional values (0.80-0.99) were explored to find more appropriate cutoff for these methods. Increasing the RMR ratio cutoff values to 0.94 for DXA and 0.92 for Cunningham 1991 yielded acceptable sensitivity of 0.80 for low $\mathrm{TT}_{3}$ values. Of the participants characterized as energy deficient using appropriate RMR ratio thresholds that yielded good-acceptable sensitivity,
Harris-Benedict and Cunningham ${ }_{1980}$ captured 87-90\% with low $\mathrm{TT}_{3}$ concentrations, while DXA and Cunningham ${ }_{1991}$ both captured $80.3 \%$ with low $\mathrm{TT}_{3}$ concentrations (Figure 4).

\section{Discussion}

This is the first paper to demonstrate that RMR ratio using various prediction equations can be applied to health outcomes that are directly related to energy deficiency, that is, low serum $\mathrm{TT}_{3}$ concentrations and severe menstrual disturbances (amenorrhea) confirmed by urinary hormone metabolites of ovarian steroids. We report that each ratio successfully distinguished between the presence or absence of energy deficiency-related conditions in exercising women, as demonstrated by significant logistic regression models for amenorrhea and low $\mathrm{TT}_{3}$. Indeed, all ratios successfully identified low $\mathrm{TT}_{3}$, despite similar energy availability values across groups. This is the first investigation to provide evidence in support of the use of RMR ratio when appropriate cutoffs are utilized as an alternative to measuring energy availability for the assessment of energetic status in exercising women.

We report that DXA and Cunningham 1991 prediction equations produced the closest estimation of measured RMR with almost $50 \%$ of predicted values falling within $\pm 100 \mathrm{kcal}$ in this 
(a) Harris-Benedict

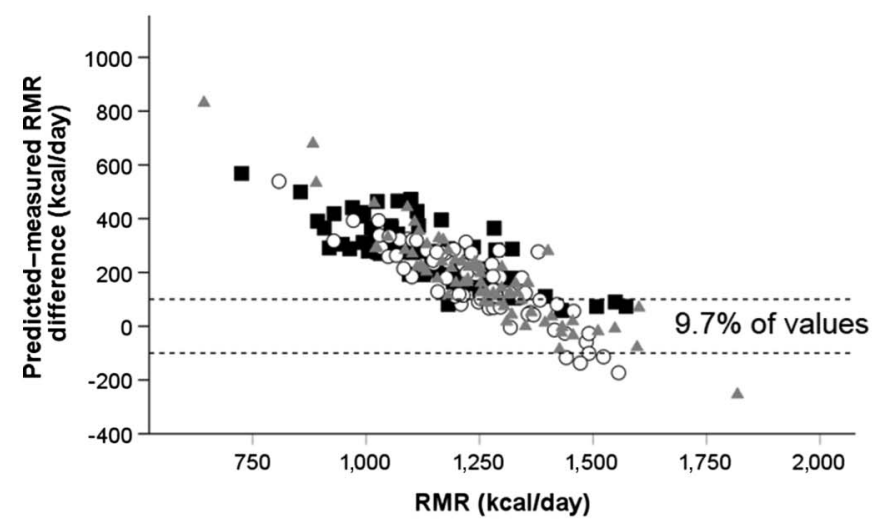

(c) Cunningham 1980

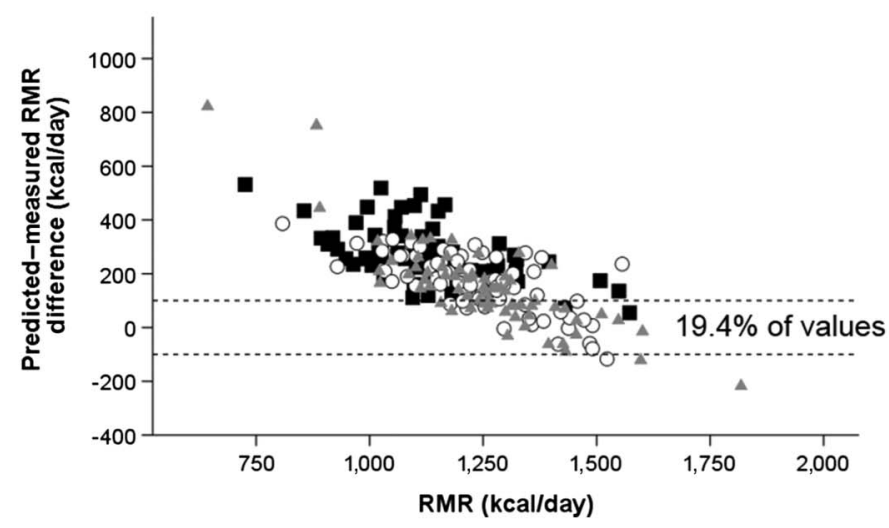

Low $\mathrm{TT}_{3}$ tertile (<1.12 nmol/l)

O Middle $\mathrm{TT}_{3}$ tertile $(1.12-1.47 \mathrm{nmol} / \mathrm{l})$

$\triangle$ High $\mathrm{TT}_{3}$ tertile (>1.47 nmol/l)

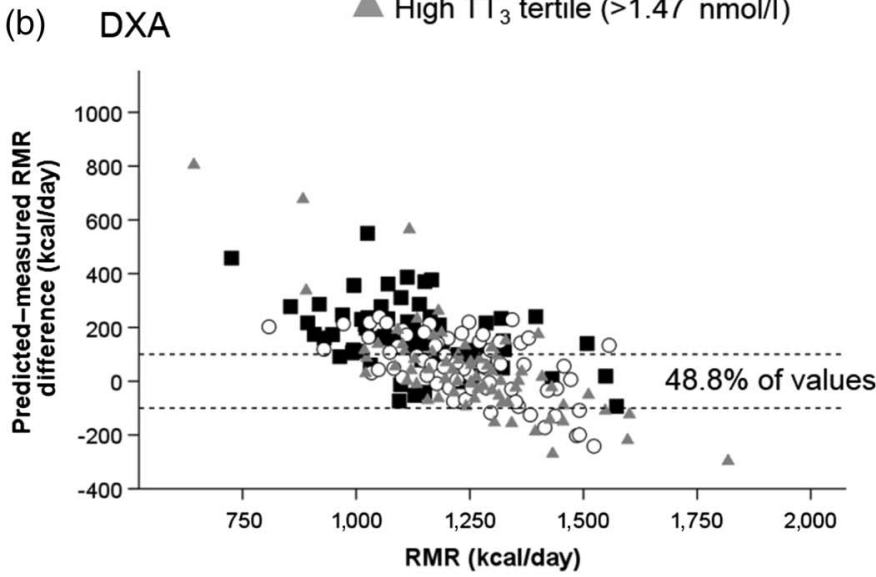

(d) Cunningham 1991

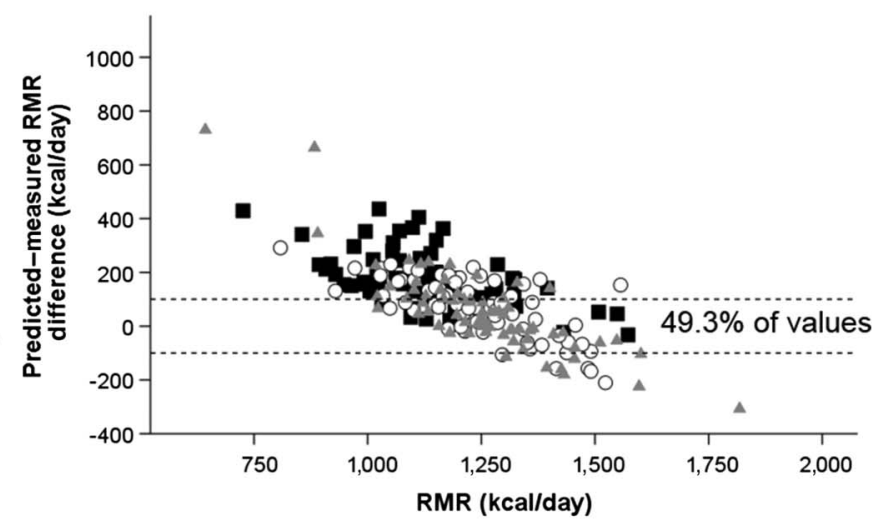

Figure 3 - Comparison of measured and predicted RMR for (a) Harris-Benedict, (b) DXA, (c) Cunningham 1980 , and (d) Cunningham 1991 methods. Dashed lines represent $\pm 100 \mathrm{kcal}$ from measured RMR for each method. Participants were grouped by $\mathrm{TT}_{3}$ tertile; black squares represent the lowest tertile, open circles represent the middle tertile, and gray triangles represent the higher tertile. Conversion factor between $\mathrm{nmol} / \mathrm{L}$ and $\mathrm{ng} / \mathrm{dl}$ is 0.0153 . $\mathrm{RMR}=$ resting metabolic rate; triiodothyronine; DXA = dual-energy X-ray absorptiometry; $\mathrm{TT}_{3}=$ total triiodothyronine .

Table 2 Outcomes of Logistic Regression Analyses Evaluating Predictors of At-Risk Triad Conditions

\begin{tabular}{|c|c|c|c|c|c|c|c|c|c|c|c|c|c|c|c|c|}
\hline \multirow[b]{2}{*}{ At-risk Triad conditions } & \multicolumn{4}{|c|}{ Harris-Benedict ratio } & \multicolumn{4}{|c|}{ DXA ratio } & \multicolumn{4}{|c|}{ Cunningham $_{1980}$ ratio } & \multicolumn{4}{|c|}{ Cunningham $_{1991}$ ratio } \\
\hline & $\boldsymbol{\beta}$ & $S E$ & $\chi^{2}$ & $p$ & $\boldsymbol{\beta}$ & $S E$ & $\chi^{2}$ & $p$ & $\boldsymbol{\beta}$ & SE & $\chi^{2}$ & $p$ & $\boldsymbol{\beta}$ & SE & $\chi^{2}$ & $p$ \\
\hline Amenorrhea & & & 6.2 & & & & 13.7 & & & & & & & & 7.8 & .005 \\
\hline Low BMI $(<18.5)$ & -4.5 & 2.3 & 3.9 & .047 & -3.4 & 2.1 & 2.7 & .097 & -4.3 & 2.4 & 3.0 & .082 & & 2.3 & 2.2 & .14 \\
\hline High drive for thinn & -2.4 & 1.7 & 2.1 & .148 & -1.3 & 1.5 & 0.7 & .400 & -2.0 & 1.8 & 1.2 & .264 & -1.7 & 1.7 & 1.0 & .310 \\
\hline Low $\mathrm{TT}_{3}(<1.12 \mathrm{nmol} / \mathrm{L})$ & -7.8 & 1.7 & 25.2 & $<.001$ & -8.6 & 1.8 & 31.9 & $<.001$ & -10.9 & 2.1 & 36.3 & $<.001$ & -10.3 & 2.0 & 36.3 & $<.001$ \\
\hline
\end{tabular}

Note. Logistic regression analyses of amenorrhea, low BMI, high drive for thinness, and low $\mathrm{TT}_{3}$. Models that indicate significant predictors of the Triad-like conditions are bolded. Conversion factor between $\mathrm{nmol} / \mathrm{L}$ and $\mathrm{ng} / \mathrm{dl}$ is 0.0153 . Cunningham $1980, \mathrm{RMR}=500+22$ (lean body mass); Cunningham $1991, \mathrm{RMR}=370+21.6$ (fat-free mass). $\mathrm{DXA}=$ dual-energy $\mathrm{X}$-ray absorptiometry; $\mathrm{BMI}=$ body mass index; $\mathrm{TT}_{3}=$ total triiodothyronine; $\mathrm{RMR}=$ resting metabolic rate.

population, most of which came from women in the high and mid $\mathrm{TT}_{3}$ tertile (i.e., energy replete). Interestingly, each method overestimated RMR, particularly when individuals had measured RMR lower than $\sim 1,200 \mathrm{kcal} /$ day. A large proportion of the participants in whom predicted RMR was overestimated (thus yielding a low $\mathrm{RMR}$ ratio) were in the lowest energetic tertile, indicated by levels of $\mathrm{TT}_{3}$ below $1.12 \mathrm{nmol} / \mathrm{L}$. This finding indicates that these prediction methods alone are not as accurate in energy-deficient women, due to metabolic adaptations leading to metabolic suppression in exercising women.

Despite the differences in predicted RMR, we have demonstrated that the RMR ratio produced by each equation have positive 
(a) Harris-Benedict

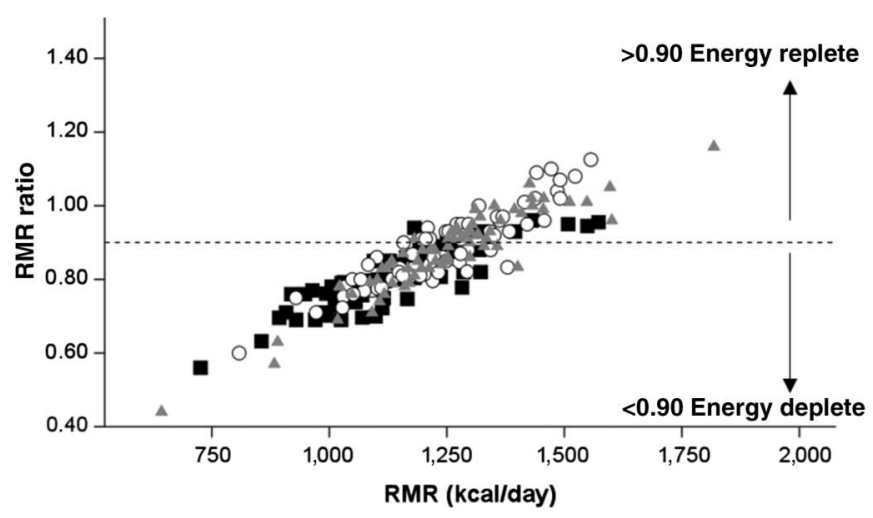

(c) Cunningham 1980

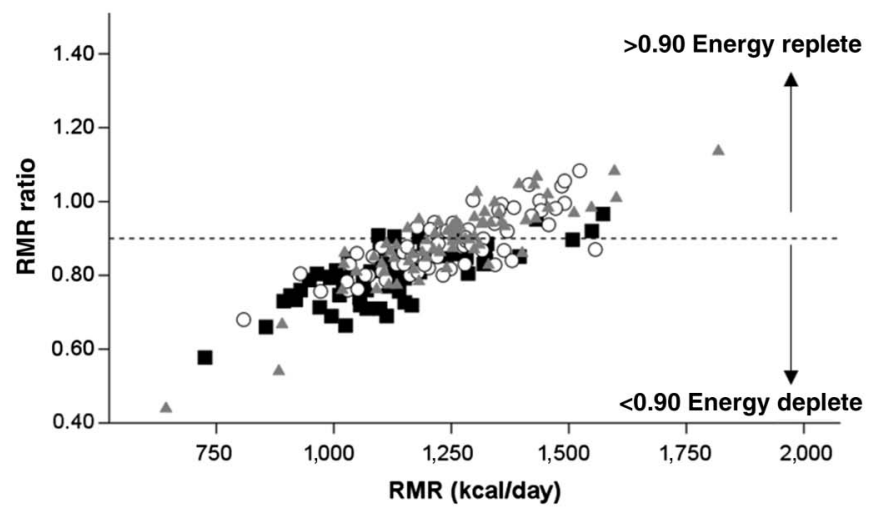

Low $\mathrm{TT}_{3}$ tertile $(<1.12 \mathrm{nmol} / \mathrm{I})$

O Middle $\mathrm{TT}_{3}$ tertile $(1.12-1.47 \mathrm{nmol} / \mathrm{l})$

$\triangle$ High $\mathrm{TT}_{3}$ tertile $(>1.47 \mathrm{nmol} / \mathrm{l})$ (b) DXA

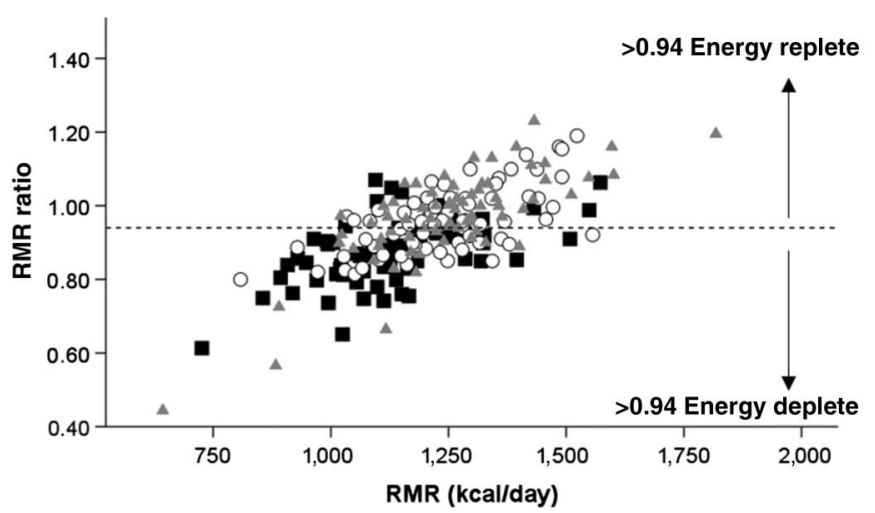

(d) Cunningham 1991

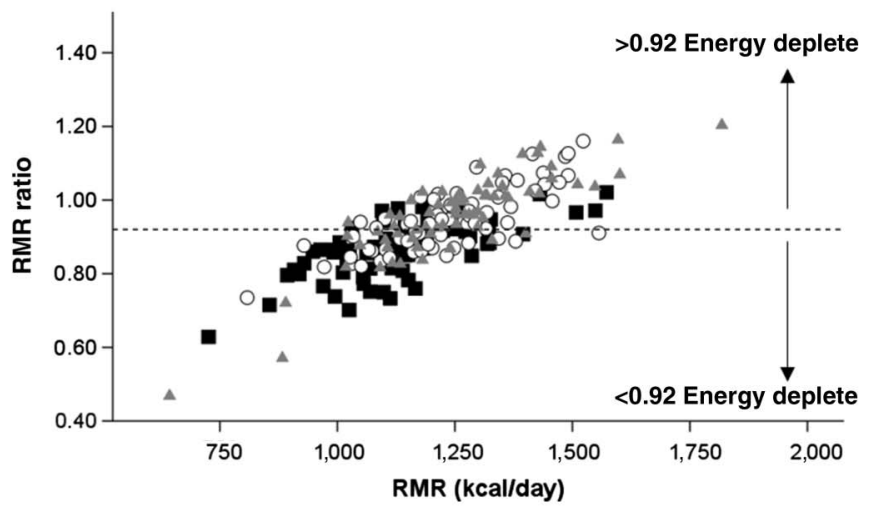

Figure 4 - Comparison of RMR ratio grouped by $\mathrm{TT}_{3}$ tertile for (a) Harris-Benedict, (b) DXA, (c) Cunningham 1980 , and (d) Cunningham 1991 methods. Dashed lines represent the ratio cutoff for each method, with values above line categorized as energy replete and values below line categorized as energy deplete. Black squares represent the lowest tertile, open circles represent the middle tertile, and gray triangles represent the higher tertile.

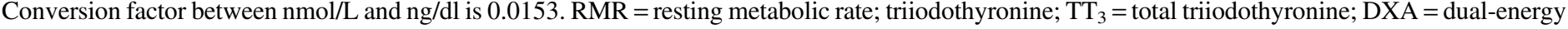
X-ray absorptiometry.

associations with $\mathrm{TT}_{3}$. Numerous studies have documented the suppression of $\mathrm{TT}_{3}$ in amenorrheic (De Souza et al., 2003; Laughlin \& Yen, 1996; Scheid et al., 2009; Vanheest et al., 2014) and energy- and estrogen-deficient women (De Souza et al., 2008; Southmayd et al., 2019), as well as in an animal model where a significant decline in circulating $\mathrm{TT}_{3}$ was coincident with the induction of amenorrhea (Williams et al., 2001). Our findings confirmed the previous report that associated DXA RMR ratio to $\mathrm{TT}_{3}$ (Koehler et al., 2016), and further demonstrates that this association is also comparable to the Harris-Benedict and Cunningham ratios.

Although a 0.90 RMR ratio cutoff, originally derived from the Harris-Benedict prediction equation (De Souza et al., 2007a, 2008), has been used with Cunningham 1980 and DXA prediction equations (Staal et al., 2018), no investigators have determined whether this universal cutoff value is suitable for all prediction equations. Our data demonstrate that in exercising women, a 0.90 RMR ratio cutoff yields the highest sensitivity for Cunningham 1980 and Harris-Benedict ratio, but not for DXA and Cunningham 1991 ratio, which may be attributed to differences in variables included in these regression equations. Because DXA and Cunningham ${ }_{1991}$ values were closer in value to measured RMR, a higher ratio cutoff is required to produce comparable sensitivity to that of HarrisBenedict and Cunningham 1980 ratio. By increasing the ratio cutoff to 0.92 for Cunningham ${ }_{1991}$ and 0.94 for DXA ratio, sensitivity increased to an acceptable level of 0.80 , while still being outside the $2-4 \%$ for the normal day-to-day variance of RMR measures (Roffey et al., 2006).

Additionally, our results demonstrate that lean body mass and fat-free mass should not be used interchangeably within the Cunningham equations, as these equations generated RMR values that were significantly different from each other. These findings indicate that previous researchers may have reported falsely elevated predicted RMR when substituting fat-free mass into the 1980 equation, which was originally intended for lean body mass (Jagim et al., 2018; Staal et al., 2018; ten Haaf \& Weijs, 2014; Thompson \& Manore, 1996; Tinsley et al., 2019). Importantly, when using fat-free mass with a Cunningham equation, the 1991 equation should be used, but a RMR ratio $>0.90$ is necessary for higher sensitivity. 
This study is limited by its cross-sectional nature and remains to be determined whether there are temporal relationships among these variables during the actual induction or reversal of menstrual disturbances. Amenorrheic women may fall across the energetic spectrum (i.e., could be in energetic recovery but have not fully recovered menstrual function) (De Souza et al., 2014) and, as such, may not be an ideal representation of energetic status, and we have been publishing this message on several occasions (De Souza et al., 2014, 2019; Southmayd et al., 2017). To address this point, we included $\mathrm{TT}_{3}$ values to provide a clinically accepted indicator of energetic status. Although RMR ratio provides an objective method of energy status estimation without cumbersome measures used to calculate energy availability, this method is limited by the need to assess RMR in a laboratory and by the use of DXA. Further investigation into mobile field measures, such as the COSMED (Rome, Italy) and Breezing (Tempe, AZ) portable metabolism tracker, need to be further evaluated and may provide a more accessible option for researchers and clinicians.

Owing to the severity of energy deficiency consequences (De Souza et al., 2007b, 2008, 2014; Nattiv et al., 2007; Williams et al., 2015) and issues with the current methods of estimating energy deficiency (i.e., energy availability) (Burke et al., 2018; Lieberman et al., 2018; Williams et al., 2015), our current investigation suggests the utility of the Harris-Benedict, DXA, and Cunningham ratio as good indicators of metabolic status. The resources available to researchers will ultimately determine the prediction method used. For example, for those without access to DXA technology, we have demonstrated the validity of the Harris-Benedict and Cunningham $_{1980}$ prediction method to identify energetically deficient individuals; for those with access to DXA and accurate measures of fat-free mass, the DXA and Cunningham ${ }_{1991}$ methods provide closer estimations to measured RMR as well as the validity to identify energetically deficient individuals. However, it should be noted that prediction equations should be used as intended (i.e., lean body mass should not be used interchangeably with fatfree mass for Cunningham equations), and a 0.90 ratio cutoff does not yield good sensitivity across all prediction methods and should be adjusted according to the method that is being utilized. Together with significant associations to hormone markers of energetic status, it may be feasible to utilize RMR ratio as an initial screening tool for energy deficiency, until a gold standard is developed and validated.

\section{Acknowledgments}

The authors would like to thank laboratory technician Ellen Bingham. The study was designed by M.J. De Souza, N.C.A. Strock, E.A. Southmayd, and K.J. Koltun. Data were collected and analyzed by N.C.A. Strock, K.J. Koltun, and E.A. Southmayd. Data interpretation and manuscript preparation were undertaken by N.C.A. Strock, K.J. Koltun, M.J. De Souza, and N.I. Williams. All authors approved the final version of the paper. Funding sources include the U.S. Department of Defense and U.S Army Medical Research and Material Command (grant no. PR054531), and seed funding for biological and life sciences provided by the PSU College of Health and Human Development. The authors have no conflict of interest to declare.

\section{References}

Altman, D.G., \& Bland, J.M. (1994). Diagnostic tests. 1: Sensitivity and specificity. British Medical Journal, 308(6943), 1552. doi:10.1136/ bmj.308.6943.1552
Bond, M.J., McDowell, A.J., \& Wilkinson, J.Y. (2001). The measurement of dietary restraint, disinhibition and hunger: An examination of the factor structure of the Three Factor Eating Questionnaire (TFEQ). International Journal of Obesity and Related Metabolic Disorders, 25(6), 900-906. PubMed ID: 11439306 doi:10.1038/sj.ijo.0801611

Burke, L.M., Lundy, B., Fahrenholtz, I.L., \& Melin, A.K. (2018). Pitfalls of conducting and interpreting estimates of energy availability in freeliving athletes. International Journal of Sport Nutrition and Exercise Metabolism, 28(4), 350-363. PubMed ID: 30029584 doi:10.1123/ ijsnem.2018-0142

Cappelleri, J.C., Bushmakin, A.G., Gerber, R.A., Leidy, N.K., Sexton, C.C., Lowe, M.R., \& Karlsson, J. (2009). Psychometric analysis of the Three-Factor Eating Questionnaire-R21: Results from a large diverse sample of obese and non-obese participants. International Journal of Obesity, 33(6), 611-620. doi:10.1038/ijo.2009.74

Cunningham, J.J. (1980). A reanalysis of the factors influencing basal metabolic rate in normal adults. American Journal of Clinical Nutrition, 33(11), 2372-2374. PubMed ID: 7435418 doi:10.1093/ajcn/33. 11.2372

Cunningham, J.J. (1991). Body composition as a determinant of energy expenditure: A synthetic review and a proposed general prediction equation. American Journal of Clinical Nutrition, 54(6), 963-969. PubMed ID: 1957828 doi:10.1093/ajen/54.6.963

De Souza, M.J., Hontscharuk, R., Olmsted, M., Kerr, G., \& Williams, N.I. (2007a). Drive for thinness score is a proxy indicator of energy deficiency in exercising women. Appetite, 48(3), 359-367. PubMed ID: 17184880 doi:10.1016/j.appet.2006.10.009

De Souza, M.J., Koltun, K.J., Strock, N.C.A., \& Williams, N.I. (2019). Rethinking the concept of an energy availability threshold and its role in the female athlete triad. Current Opinions in Physiology, 10, $35-42$.

De Souza, M.J., Lee, D.K., VanHeest, J.L., Scheid, J.L., West, S.L., \& Williams, N.I. (2007b). Severity of energy-related menstrual disturbances increases in proportion to indices of energy conservation in exercising women. Fertility and Sterility, 88(4), 971-975. PubMed ID: 17418159 doi:10.1016/j.fertnstert.2006.11.171

De Souza, M.J., Miller, B.E., Loucks, A.B., Luciano, A.A., Pescatello, L.S., Campbell, C.G., \& Lasley, B.L. (1998). High frequency of luteal phase deficiency and anovulation in recreational women runners: blunted elevation in follicle-stimulating hormone observed during luteal-follicular transition. The Journal of Clinical Endocrinology \& Metabolism, 83(12), 4220-4232. PubMed ID: 9851755

De Souza, M.J., Nattiv, A., Joy, E., Misra, M., Williams, N.I., Mallinson, R.J., . . . Expert Panel. (2014). 2014 female athlete triad coalition consensus statement on treatment and return to play of the female athlete triad: 1st International Conference held in San Francisco, California, May 2012 and 2nd international conference held in Indianapolis, Indiana, May 2013. British Journal of Sports Medicine, 48(4), 289. PubMed ID: 24463911 doi:10.1136/bjsports-2013093218

De Souza, M.J., Toombs, R.J., Scheid, J.L., O’Donnell, E., West, S.L., \& Williams, N.I. (2010). High prevalence of subtle and severe menstrual disturbances in exercising women: Confirmation using daily hormone measures. Human Reproduction, 25(2), 491-503. PubMed ID: 19945961 doi:10.1093/humrep/dep411

De Souza, M.J., Van Heest, J., Demers, L.M., \& Lasley, B.L. (2003). Luteal phase deficiency in recreational runners: Evidence for a hypometabolic state. The Journal of Clinical Endocrinology \& Metabolism, 88(1), 337-346. PubMed ID: 12519874 doi:10.1210/ jc. $2002-020958$

De Souza, M.J., West, S.L., Jamal, S.A., Hawker, G.A., Gundberg, C.M., $\&$ Williams, N.I. (2008). The presence of both an energy deficiency 
and estrogen deficiency exacerbate alterations of bone metabolism in exercising women. Bone, 43(1), 140-148. PubMed ID: 18486582 doi:10.1016/j.bone.2008.03.013

Elia, M. (1992). Organ and tissue contributions to metabolic rate. In J.M. Kinney and H.N. Tucker, Eds. Energy Metabolism: Tissue Determinants and Cellular Corollaries. New York, NY: Raven Press, pp. 61-80.

Gallagher, D., Belmonte, D., Deurenberg, P., Wang, Z., Krasnow, N., Pi-Sunyer, F.X., \& Heymsfield, S.B. (1998). Organ-tissue mass measurement allows modeling of REE and metabolically active tissue mass. American Journal of Physiology, 275(2, Pt. 1), E249-E258.

Garner, D. (1991). Eating disorder inventory-2 manual. Odessa, FL: Psychological Assessment Resources.

Gibbs, J.C., Williams, N.I., Scheid, J.L., Toombs, R.J., \& De Souza, M.J. (2011). The association of a high drive for thinness with energy deficiency and severe menstrual disturbances: Confirmation in a large population of exercising women. International Journal of Sport Nutrition and Exercise Metabolism, 21(4), 280-290. PubMed ID: 21813911 doi:10.1123/ijsnem.21.4.280

Harris, J.A., \& Benedict, F.G. (1918). A biometric study of human basal metabolism. Proceedings of the National Academy of Sciences of the United States of America, 4(12), 370-373. PubMed ID: 16576330 doi:10.1073/pnas.4.12.370

Hayes, M., Chustek, M., Wang, Z., Gallagher, D., Heshka, S., Spungen, A., . . . Heymsfield, S.B. (2002). DXA: Potential for creating a metabolic map of organ-tissue resting energy expenditure components. Obesity Research, 10(10), 969-977. PubMed ID: 12376576 doi:10.1038/oby.2002.132

Jagim, A.R., Camic, C.L., Kisiolek, J., Luedke, J., Erickson, J., Jones, M.T., \& Oliver, J.M. (2018). Accuracy of resting metabolic rate prediction equations in athletes. Journal of Strength and Conditioning Research, 32(7), 1875-1881. PubMed ID: 28682934 doi:10. 1519/JSC.0000000000002111

Koehler, K., Williams, N.I., Mallinson, R.J., Southmayd, E.A., Allaway, H.C., \& De Souza, M.J. (2016). Low resting metabolic rate in exercise-associated amenorrhea is not due to a reduced proportion of highly active metabolic tissue compartments. American Journal of Physiology_Endocrinology and Metabolism, 311(2), E480-E487. PubMed ID: 27382033 doi:10.1152/ajpendo.00110.2016

Koltun, K.J., De Souza, M.J., Scheid, J.L., \& Williams, N.I. (2019). MON-111 amenorrhea in exercising women is associated with a greater reduction in post-prandial ghrelin. Journal of the Endocrine Society, 3(Suppl. 1), April-May 2019, MON-111, https://doi.org/10. 1210/js.2019-MON-111.

Laughlin, G.A., \& Yen, S.S. (1996). Nutritional and endocrine-metabolic aberrations in amenorrheic athletes. The Journal of Clinical Endocrinology \& Metabolism, 81(12), 4301-4309. PubMed ID: 8954031

Leibel, R.L., Rosenbaum, M., \& Hirsch, J. (1995). Changes in energy expenditure resulting from altered body weight. New England Journal of Medicine, 332(10), 621-628. PubMed ID: 7632212 doi:10. 1056/NEJM199503093321001

Lieberman, J.L., De Souza, M.J., Wagstaff, D.A., \& Williams, N.I. (2018). Menstrual disruption with exercise is not linked to an energy availability threshold. Medicine \& Science in Sports \& Exercise, 50(3), 551-561. doi:10.1249/MSS.0000000000001451

Loucks, A.B., \& Heath, E.M. (1994). Induction of low-T3 syndrome in exercising women occurs at a threshold of energy availability. American Journal of Physiology, 266(3, Pt. 2), R817-R823. PubMed ID: 8160876

Loucks, A.B., \& Thuma, J.R. (2003). Luteinizing hormone pulsatility is disrupted at a threshold of energy availability in regularly menstruating women. The Journal of Clinical Endocrinology \& Metabolism, 88(1), 297-311. PubMed ID: 12519869 doi:10.1210/ jc.2002-020369

Loucks, A.B., Verdun, M., \& Heath, E.M. (1998). Low energy availability, not stress of exercise, alters LH pulsatility in exercising women. Journal of Applied Physiology, 84(1), 37-46. PubMed ID: 9451615 doi:10.1152/jappl.1998.84.1.37

Melchior, J.C., Rigaud, D., Rozen, R., Malon, D., \& Apfelbaum, M. (1989). Energy expenditure economy induced by decrease in lean body mass in anorexia nervosa. European Journal of Clinical Nutrition, 43(11), 793-799. PubMed ID: 2627927

Melin, A., Tornberg, A.B., Skouby, S., Moller, S.S., Sundgot-Borgen, J., Faber, J., . . Sjodin, A. (2015). Energy availability and the female athlete triad in elite endurance athletes. Scandinavian Journal of Medicine \& Science in Sports, 25(5), 610-622. PubMed ID: 24888644 doi:10.1111/sms.12261

Morley, J.E., Slag, M.F., Elson, M.K., \& Shafer, R.B. (1983). The interpretation of thyroid function tests in hospitalized patients. Journal of the American Medical Association, 249(17), 2377-2379. PubMed ID: 6403725 doi:10.1001/jama.1983.03330410063031

Nattiv, A., Loucks, A.B., Manore, M.M., Sanborn, C.F., Sundgot-Borgen, J., Warren, M.P., \& American College of Sports Medicine. (2007). American College of Sports Medicine position stand: The female athlete triad. Medicine \& Science in Sports \& Exercise, 39(10), 18671882. doi:10.1249/mss.0b013e318149f111

O’Donnell, E., Harvey, P.J., Goodman, J.M., \& De Souza, M.J. (2007). Long-term estrogen deficiency lowers regional blood flow, resting systolic blood pressure, and heart rate in exercising premenopausal women. American Journal of Physiology-Endocrinology and Metabolism, 292(5), E1401-E1409. doi:10.1152/ajpendo.00547. 2006

Reed, J.L., De Souza, M.J., Mallinson, R.J., Scheid, J.L., \& Williams, N.I. (2015). Energy availability discriminates clinical menstrual status in exercising women. Journal of the International Society of Sports Nutrition, 12, 11. PubMed ID: 25722661 doi:10.1186/s12970-0150072-0

Roffey, D.M., Byrne, N.M., \& Hills, A.P. (2006). Day-to-day variance in measurement of resting metabolic rate using ventilated-hood and mouthpiece \& nose-clip indirect calorimetry systems. Journal of Parenteral and Enteral Nutrition, 30(5), 426-432. PubMed ID: 16931612 doi: $10.1177 / 0148607106030005426$

Santoro, N., Crawford, S.L., Allsworth, J.E., Gold, E.B., Greendale, G.A., Korenman, S., . . . Weiss, G. (2003). Assessing menstrual cycles with urinary hormone assays. American Journal of PhysiologyEndocrinology and Metabolism, 284(3), E521-E530. PubMed ID: 12441312 doi:10.1152/ajpendo.00381.2002

Scheid, J.L., Williams, N.I., West, S.L., VanHeest, J.L., \& De Souza, M.J. (2009). Elevated PYY is associated with energy deficiency and indices of subclinical disordered eating in exercising women with hypothalamic amenorrhea. Appetite, 52(1), 184-192. PubMed ID: 18929607 doi:10.1016/j.appet.2008.09.016

Southmayd, E.A., Mallinson, R.J., Williams, N.I., Mallinson, D.J., \& De Souza, M.J. (2017). Unique effects of energy versus estrogen deficiency on multiple components of bone strength in exercising women. Osteoporosis International, 28(4), 1365-1376. PubMed ID: 28032184 doi:10.1007/s00198-016-3887-x

Southmayd, E.A., Williams, N.I., Mallinson, R.J., \& De Souza, M.J. (2019). Energy deficiency suppresses bone turnover in exercising women with menstrual disturbances. The Journal of Clinical Endocrinology \& Metabolism, 104(8), 3131-3145.

Staal, S., Sjodin, A., Fahrenholtz, I., Bonnesen, K., \& Melin, A.K. (2018). Low RMR ratio as a surrogate marker for energy deficiency, the 
choice of predictive equation vital for correctly identifying male and female ballet dancers at risk. International Journal of Sport Nutrition and Exercise Metabolism, 28(4), 412-418. PubMed ID: 29405782 doi:10.1123/ijsnem.2017-0327

ten Haaf, T., \& Weijs, P.J. (2014). Resting energy expenditure prediction in recreational athletes of 18-35 years: Confirmation of Cunningham equation and an improved weight-based alternative. PLoS One, 9(9), e108460. PubMed ID: 25275434 doi:10.1371/journal.pone. 0108460

Thompson, J., \& Manore, M.M. (1996). Predicted and measured resting metabolic rate of male and female endurance athletes. Journal of the American Dietetic Association, 96(1), 30-34. PubMed ID: 8537566 doi:10.1016/S0002-8223(96)00010-7

Thomson, J.E., Baird, S.G., \& Thomson, J.A. (1977). Thyroid function in dietary amenorrhoea. Clinical Endocrinology, 7(5), 383-388. PubMed ID: 589804 doi:10.1111/j.1365-2265.1977.tb03347.x

Tinsley, G.M., Graybeal, A.J., \& Moore, M.L. (2019). Resting metabolic rate in muscular physique athletes: Validity of existing methods and development of new prediction equations. Applied Physiology, Nutrition, and Metabolism, 44(4), 397-406. PubMed ID: 30240568 doi:10.1139/apnm-2018-0412

Vaisman, N., Rossi, M.F., Goldberg, E., Dibden, L.J., Wykes, L.J., \& Pencharz, P.B. (1988). Energy expenditure and body composition in patients with anorexia nervosa. Journal of Pediatrics, 113(5), 919-924. PubMed ID: 3183853 doi:10.1016/S0022-3476(88) $80032-5$

Vanheest, J.L., Rodgers, C.D., Mahoney, C.E., \& De Souza, M.J. (2014). Ovarian suppression impairs sport performance in junior elite female swimmers. Medicine \& Science in Sports \& Exercise, 46(1), 156166. PubMed ID: 23846160 doi:10.1249/MSS.0b013e3182a32b72
Vescovi, J.D., Scheid, J.L., Hontscharuk, R., \& De Souza, M.J. (2008). Cognitive dietary restraint: Impact on bone, menstrual and metabolic status in young women. Physiology \& Behavior, 95(1-2), 48-55. PubMed ID: 18508099 doi:10.1016/j.physbeh. 2008.04.003

Wade, G.N., Schneider, J.E., \& Li, H.Y. (1996). Control of fertility by metabolic cues. American Journal of Physiology, 270(1, Pt. 1), E1-E19. PubMed ID: 8772468

Williams, N.I., Caston-Balderrama, A.L., Helmreich, D.L., Parfitt, D.B., Nosbisch, C., \& Cameron, J.L. (2001). Longitudinal changes in reproductive hormones and menstrual cyclicity in cynomolgus monkeys during strenuous exercise training: Abrupt transition to exerciseinduced amenorrhea. Endocrinology, 142(6), 2381-2389. PubMed ID: 11356685 doi:10.1210/endo.142.6.8113

Williams, N.I., Leidy, H.J., Hill, B.R., Lieberman, J.L., Legro, R.S., \& De Souza, M.J. (2015). Magnitude of daily energy deficit predicts frequency but not severity of menstrual disturbances associated with exercise and caloric restriction. American Journal of PhysiologyEndocrinology and Metabolism, 308(1), E29-E39. PubMed ID: 25352438 doi:10.1152/ajpendo.00386.2013

Williams, N.I., Mallinson, R.J., \& De Souza, M.J. (2019). Rationale and study design of an intervention of increased energy intake in women with exercise-associated menstrual disturbances to improve menstrual function and bone health: The REFUEL Study. Contemporary Clinical Trials Communications, 14, 100325.

Williams, N.I., Young, J.C., McArthur, J.W., Bullen, B., Skrinar, G.S., \& Turnbull, B. (1995). Strenuous exercise with caloric restriction: Effect on luteinizing hormone secretion. Medicine \& Science in Sports \& Exercise, 27(10), 1390-1398. doi:10.1249/00005768199510000-00007 